\title{
Capacidade Produtiva e Análise De Custo Em Uma Empresa No Setor De Confecção
}

\author{
João Paulo Pereira \\ Graduação em Ciências Contábeis pela Faculdade Estácio de Sá \\ Analista contábil na empresa Isopasse \\ Rua Dona Leonildes Celho, 566. Praia Comprida. São José/SC. CEP: 88103-660 \\ E-mail: j-paulo-p@hotmail.com \\ Marcelo Antonio Pierri Junior \\ Mestrado em Contabilidade pela Universidade Federal de Santa Catarina - \\ UFSC \\ Professor no Centro Universitário Estácio de Sá/Santa Catarina \\ Avenida Atilio Pedro Pagani 381, apto 501. Passa Vinte. Palhoça/SC. CEP: \\ 88132-149 \\ E-mail: marcelopierrijr@gmail.com
}

\section{RESUMO}

O processo de análise dos custos contempla a averiguação dos departamentos, no qual cada um deles é analisado individualmente por meio de observação dos processos envolvendo colaboradores, medindo o tempo médio gasto por unidade produzida e o custo de cada etapa. O objetivo deste estudo é analisar a capacidade produtiva por etapa de cada setor de uma empresa têxtil. Este artigo é um estudo de caso sobre uma indústria de confecção com mais de 22 anos de mercado, sua linha de produtos consiste em artigos de bens duráveis para passadoria e lavanderia. $O$ estudo é baseado nos custos de produção, capacidade produtiva por departamento e caminho lógico dos produtos durante a produção. A empresa divide seu setor produtivo em três departamentos onde foram estudados os seus processos de produção, com base nos custos e no tempo. Dentre os resultados, comparando a capacidade produtiva diária dos setores, pode-se constatar que, na categoria setor de corte, consegue-se produzir uma média de 3 vezes mais do que o setor de costura, em relação ao forro para tábua, é uma média de 6 vezes mais do que o setor de costura, já, nos protetores, chega a 5 vezes mais produção do setor de corte em relação ao de costura. Dessa forma, através desta pesquisa técnica podese analisar e remodelar os processos nos setores com base na capacidade produtiva e nos custos por processo.

Palavras-Chave: Custo por Departamentalização. Indústria Têxtil. Capacidade Produtiva. 


\title{
Productive Capacity and Cost Analysis in a Clothing Industry Company
}

\begin{abstract}
The cost analysis process contemplates the investigation of the departments, in which each one of them is analyzed individually through the observation of processes involving employees, measuring the average time spent per unit produced and the cost of each step. The objective of this study is to analyze the productive capacity by stage of each sector of a textile company. This article is a case study about a clothing industry with over 22 years of market, its product line consists of durable goods items for ironing and laundry. The study is based on production costs, production capacity by department, and logical path of products during production. The company divides its production sector into three departments, where its production processes were studied, based on costs and time. Among the results, comparing the daily productive capacity of the sectors, it can be seen that in the cutting sector category it can produce an average of 3 times more than in the sewing sector. In relation to the board lining, it is an average of 6 times more than the sewing sector. In protectors it reaches 5 times more production in the cutting sector than in the sewing sector. Thus, through this technical research it is possible to analyze and remodel the processes in the sectors based on the productive capacity and costs per process.
\end{abstract}

Keywords: Cost per Departmentalization. Textile industry. Productive capacity.

\section{Análisis de capacidad productiva y costos en una empresa del sector de} confección

\section{RESUMEN}

El proceso de análisis de costos comienza con la investigación de los departamentos, en la que cada uno de ellos es analizado individualmente a través de la observación de procesos que involucran a los empleados, midiendo el tiempo promedio empleado por unidad producida y el costo de cada paso. El objetivo de este estudio es analizar la capacidad productiva por etapa de cada sector de una empresa textil. Este artículo es un caso de estudio sobre una industria de la confección con más de 22 años de mercado, su línea de productos consiste en artículos de bienes duraderos para planchar y lavar la ropa. El estudio se basa en los costos de producción, la capacidad de producción por departamento y la ruta lógica de los productos durante la producción. La empresa divide su sector productivo en tres departamentos, donde se estudiaron sus procesos productivos, en base a costos y tiempo. Entre los resultados, al comparar la capacidad productiva diaria de los sectores, se puede observar que en la categoría de sector de corte puede producir un promedio de 3 veces más que el sector de costura, en relación al forro de tableros, es un promedio de 6 veces más que el sector de la costura, en protectores alcanza 5 veces más producción en el sector del corte que en el sector de la costura. Así, a través de esta investigación técnica es posible analizar y 
remodelar los procesos en los sectores en función de la capacidad productiva y en los costos por proceso.

Palabras clave: Costo por Departamentalización. Industria textil. Capacidad productiva.

\section{INTRODUÇÃO}

As decisões devem estar cada vez mais apoiadas por instrumentos capazes de fornecer informações que impulsionem as organizações a cumprir sua missão e assegurar sua longevidade no mercado (Raupp \& Beuren, 2014). Uma série de mudanças, como a globalização econômica, os avanços tecnológicos, a tecnologia da informação, entre outros, impulsionou as empresas dos mais diversos setores a reformularem suas atividades operacionais. Além disso, procuram permanecer em processo contínuo de aprimoramento para poder resistir as diversas mudanças impostas pelo mercado e pela concorrência acirrada entre empresas (Coronetti, Beuren, \& Sousa, 2003).

Assim, por conta desse ambiente tão acirrado em que as indústrias estão inseridas, surge a necessidade de as empresas compreenderem os seus custos com o objetivo de administrar a sua capacidade de produção e gerar riqueza para conduzir seus negócios da forma mais abrangente possível, encontrando uma maneira de estar à frente da concorrência (Rabelo, Borgert, \& Medeiros, 2011). Tal situação, requer das indústrias um controle preciso das atividades fabril, usando instrumentos rápidos e eficientes para o aprimoramento de seus métodos de custeio dos produtos e serviços de gerenciamento de resultados e de apoio às decisões (Braga, 2009).

No contexto, para permanecerem no mercado competitivo, as organizações têm a necessidade de estarem sempre preparadas para combater a concorrência, sem perder a qualidade e mantendo a lucratividade, de forma que possam ter um sistema de custeio e uma ferramenta de auxílio para manter uma eficácia e eficiência, não somente na indústria, mas também no comércio e serviços (Barsanti, 2016). 
Seguindo tais pressupostos, tem-se que a indústria de confecção, por ser um ramo industrial baseado no uso intensivo de mão de obra, ao qual poucas inovações técnicas, limitando-se à máquina de costura industrial e ao trabalho manual (Araújo, 2002).

Conforme ABIT (2015), "o setor têxtil reúne mais de 33 mil empresas (com mais de 5 funcionários) das quais mais de $80 \%$ são confecções de pequeno e médio porte, em todo o território nacional".

Por conta disso, surge a necessidade de conhecer os custos que abrangem a cadeia produtiva, visto que grande parte deles é representada pela mão de obra direta que é considerada "custo variável". Assim, para que possam ser avaliados os efeitos marginais da venda de uma unidade, considera-se um custo fixo, já que não varia a cada unidade que se venda ou que se deixe de vender (Sá, 2011).

Visando o cenário explicitado, tem-se o seguinte problema de pesquisa: Qual a capacidade produtiva por departamento com base nos custos de uma indústria do setor de confecção? Para responder o questionamento o objetivo geral é analisar a capacidade produtiva por etapa de cada setor de uma empresa têxtil. O estudo é realizado em uma indústria do setor de confecção situada em Santa Catarina. A empresa é intitulada como Alfa por questão de sigilo. Com mais de 22 anos de mercado, responsável pela produção de artigos para o bazar, na linha de lavanderia e passadoria. A pesquisa justifica-se neste setor pelo aumento da competitividade decorrente do mercado exterior, principalmente o asiático, que exigiu da empresa um estudo sobre os processos de custos de fabricação dos produtos para a tomada de decisão nesse ambiente competitivo.

Conforme dos dados estatísticos fornecidos pela Associação Brasileira da Indústria Têxtil e de Confecção (ABIT), de janeiro a março de 2019, a indústria de transformação apresentou queda de 1,4\%, o segmento têxtil uma queda de $3,4 \%$ e o de confecção $4,3 \%$, demonstrando em relatórios detalhados que as empresas de países estrangeiros vêm consumindo parte do mercado de exportação brasileiro.

O processo de análise dos custos se inicia com a averiguação dos departamentos, no qual cada um deles é analisado individualmente por meio de observação dos processos envolvendo colaboradores, medindo o tempo médio gasto por unidade produzida e o custo de cada etapa. O caminho lógico que o 
produto percorre na linha de produção, da entrada de insumos no estoque até a saída dos produtos acabados. Ainda, o trabalho abrange quatro categorias com produtos fabricados por Alfa de diferentes tamanhos, modelos e cores, tendo um total de 19 códigos de barras, identificados pelo EAN, European Article Number, sendo utilizado para a identificação direta do produto.

Para a obtenção dos resultados através de análises junto aos documentos pertinentes da empresa Alfa, utilizou-se a ferramenta Microsoft Excel, no qual se obteve os elementos para a formação dos custos, junto com os dados para a complementação dos objetivos secundários. Como tópicos específicos, o estudo tem como finalidade o mapeamento dos processos produtivos de cada departamento, buscando mensurar a capacidade produtiva, entre os setores da empresa Alfa, medindo o desemprenho apresentado, a capacidade máxima de produção diária, o tempo médio de produção e a distribuição dos colaboradores nas etapas do processo.

Em sequência, com a compreensão dos custos que a empresa Alfa apresenta, esta pesquisa buscar encontrar uma nova maneira mais eficiente para aplicar o custo de produção, de forma a identificar ponto máximo da capacidade produtiva da empresa, identificando no processo possíveis gargalos produtivos e a capacidade de produção dos departamentos. Desse modo, esta pesquisa poderá mudar o costume das tomadas de decisões estratégicas e organizacionais, contribuindo para os gestores uma melhor forma de usar os recursos.

\section{REFERENCIAL TEÓRICO}

A contabilidade de custos é um ramo da contabilidade que produz informações relevantes para uma entidade, sendo de importância para auxiliar as tomadas de decisão, a apuração das informações e alocação dos custos de produção de uma entidade, de forma a fornecer dados detalhados sobre custos que a gestão precisa para controlar as operações atuais e planejar para o futuro (Derbeck \& Nagy, 2001). 
Assim, tem-se que o custo é um instrumento essencial para uma empresa e está presente em diversas etapas do processo de produção de um produto ou serviço.

Nesse sentido, Koliver (2009) aborda que "os custos correspondem ao valor de mutação patrimonial qualitativa, ocorrida no ciclo operacional interno de uma entidade". Partindo dessa definição, tem-se que toda mudança da entidade em transformar ativos em outros ativos, como insumos em produto acabado, está ligado diretamente à contabilidade de custos, tendo o dever de manter uma eficiência para a obtenção de valores diretos e indiretos que afetam na criação do valor de produtos vendidos ou serviços prestados (Martins, 2010).

Para tanto, o estudo dos conceitos e terminologias de custos é fundamental para o entendimento da contabilidade de custos e gestão de custos, de acordo com Santos (2018), o entendimento dos sistemas de informações que os produzem, sendo que entre eles, existe a definição em custos fixos e variáveis. Levando em consideração a relação entre o valor total de um custo e o volume de atividade numa unidade de tempo (Oliveira, 2000).

De tal modo, os custos fixos são aqueles que não variam no aumento ou diminuição da quantidade produzida e tendem a permanecerem constantes nas variações da cadeia produtiva. Diferente dos custos variáveis que variam diretamente com as modificações no nível de produtividade, sendo o inverso dos custos fixos, quanto maior a produção maior os custos variáveis (Martins, 2010).

Há, também, a classificação de custos em diretos e indiretos sendo útil para apropriação aos objetos de custeio, cuja finalidade é a realização de análises de rentabilidade, controle de gastos, formação de preço entre outros (Santos, 2018).

Martins (2010) aborda por custos diretos aqueles atribuídos diretamente no produto ou objeto de custeio, são de fácil identificação por serem custos que afetam diretamente a produção, como matéria-prima, mão de obra, entre outros. Por serem custos diretos os seus valores já estão alocados no produto e não se tem a necessidade de rateio. Porém, os custos diretos por natureza que são tratados de maneira diferente, como indiretos em função de sua irrelevância ou da dificuldade de sua medição, ou até do interesse da empresa em ser mais ou menos rigorosa em suas informações (Oliveira, 2000). 
Já, por custos indiretos são aqueles cuja identificação ao objeto de custo é feita de forma indireta, mediante parâmetros estabelecidos previamente; portanto, não podem ser quantificados nos portadores finais (Santos, 2018). São alocados ao objeto de custeio por meio de critérios de rateio. A escolha por diferentes critérios de rateios pode mudar o custo de determinado bem, sem que, obrigatoriamente, tenham acontecido alterações no processo produtivo (Martins, 2010).

Nas indústrias, a grande maioria opera por sistema departamental, e além de vários produtos fabricados, existem diversos departamentos no qual eles passam. Segundo Alonso (1999), a departamentalização consiste em dividir a indústria em segmentos, intitulados como departamentos. Assim, um departamento é representado na prática por homens e máquinas, e na maioria das vezes se identifica com um centro de custos, onde nele são acumulados os custos indiretos para posterior alocação aos produtos (Santos, 2018).

Assim, como a indústria divide os setores produtivos em departamentos, apura-se não só custos dos produtos, mas também custos por departamento, medindo dessa forma a capacidade de cada centro de custos, a sua influência na cadeia produtiva (Santos, 2018).

De acordo com Alonso (1999), os departamentos podem ser divididos em dois grupos: departamentos de produção e departamentos de serviço. Onde os departamentos de produção são aqueles que atuam sobre os produtos e tem seus custos apropriados diretamente a esses, e os departamento de serviço não atuam diretamente na produção e sua finalidade é de prestar serviços aos departamentos de produção. Seus custos não são apropriados aos produtos, pois esses não transitam por eles, e sim transferidos para os departamentos de produção que se beneficiam dos serviços deles.

Sobre os métodos de custeio, eles podem ser utilizados tanto pelas organizações industriais, quanto pelas comerciais e prestadoras de serviços, sendo que esses métodos são utilizados para determinar o valor dos objetos de custeio; reduzir custos, melhorar os processos; eliminar desperdícios; decidir entre produzir ou terceirizar; e eliminar, criar e aumentar, ou diminuir, a linha de produção de certos produtos, entre muitos outros (Abbas, Gonçalves, \& Leoncine, 2012). 
Dentre esses métodos, têm-se o custeio por absorção com departamentalização, segundo Martins (2010), é um preceito onde se apropriam todos os custos de produção quer fixos, quer variáveis, quer diretos ou indiretos, são absorvidos pelos produtos e considerados para fins de valorização dos estoques. Entretanto, apenas os gastos genéricos não ligados à fabricação não são absorvidos pelos produtos, tal como os administrativos, financeiros e de vendas (Santos 2018).

Segundo Martins (2010), o custeio por absorção pode ser realizado primeiramente pela separação entre custo e despesas, visto que as despesas não podem ser alocadas aos produtos, pois pertencem ao período em que incorrem. $O$ segundo ponto a ser observado, diz respeito à apropriação dos custos diretos, por meio da identificação dos custos que estão diretamente relacionados com os produtos. Por fim, tem-se a apropriação dos custos indiretos, por meio de bases de rateio, já que esses custos não são identificáveis diretamente aos produtos. Importante destacar que o método de custeio por absorção separa os custos e despesas que estão envolvidos no processo de fabricação, lembrando que as despesas não podem distribuídas nos produtos e com base no rateio alguns custos não poderão ser identificados de forma direta aos produtos ou serviços.

Assim, diante de todo o exposto, essas ferramentas ajudam na identificação e descrição das relações entre capacidade de produção. Para a construção de uma rede de relações de causa e efeito que terá um impacto sobre o desempenho produtivo da indústria, com enfoque na quantidade produzida e no tempo utilizado, usando como base os custos dos departamentos sob análise. Determinar essa rede é um passo essencial para a análise diagnóstica do problema de pesquisa.

\section{METODOLOGIA}

Para a realização desta pesquisa foram utilizados dados secundários e primários. A pesquisa é caracterizada como documental e qualitativa.

A pesquisa quanto aos procedimentos técnicos pode ser classificada como estudo de caso. Optou-se na escolha de uma indústria no setor de confecção, uma empresa de pequeno porte optante pelo Simples Nacional, com mais de 22 anos de mercado. 
A empresa possui atualmente dois sócios com uma participação igualitária, conta com sete colaboradores na fábrica, dois na parte administrativa e um vendedor externo, totalizando 12 colaboradores. Como a empresa em questão solicitou sigilo sobre a sua identidade, reserva-se neste artigo o direito de ocultar o nome da referida indústria, que é designada como "Alfa".

A Empresa fabrica produtos de utilidade doméstica, atualmente comercializa os seus produtos em supermercados, lojas varejistas do Sul do país, e por meio do marketplace e e-commerce próprio. Sua linha de produtos consiste em artigos de bens duráveis para passadoria e lavanderia, como almofadas, forros para passar roupa, protetor para lavar roupa e capa de máquina. Os produtos possuem cores, tamanhos diferentes e são contabilizados por EAN. No Quadro 01 apresentam-se os dados dos produtos.

\begin{tabular}{|c|c|c|c|}
\hline Produto & Cor & Tamanhos & EAN \\
\hline Capa de Máquina & 3 & 5 & 11 \\
\hline Forro P/ Roupa & 1 & 8 & 8 \\
\hline Protetor P/ Roupa & 1 & 4 & 4 \\
\hline Almofada & 1 & 2 & 2 \\
\hline
\end{tabular}

\section{Quadro 01. Quantidade de Produtos.}

Fonte: Dados da Pesquisa, 2019.

A empresa tem três documentos de estudos realizados anteriormente que auxiliaram na quantificação e apuração dos dados, entre eles são: (I) proposta de um plano estratégico para a empresa, (II) proposta de um sistema logístico para o comércio eletrônico da empresa (III) plano de negócio: valuation da empresa. Foi feita uma pesquisa nos arquivos e dados da empresa. Para a tabulação dos dados utiliza-se o Microsoft Excel.

A empresa Alfa divide seu setor produtivo em três setores/departamentos, corte, costura e embalagem que são intitulados pela empresa como etapas, sendo que alguns colaboradores são remanejados de acordo com a necessidade de cada etapa. Para definir um valor para cada setor, utilizou-se o método de custeio por absorção, que consiste na verificação de todos os custos implicam na produção dos bens, fixos ou variáveis (Sá, 2011). Utilizando os custos de produção diretos e os 
custos indiretos, também são rateados dentro do custo do produto seguindo o critério estabelecido pela empresa (Sato, 2008). O método foi utilizado para verificação de quais custos a empresa apresenta em cada setor.

Para levantamento dos custos foram apurados dados fornecidos pela empresa Alfa e a partir da coleta e análise de alguns pontos das etapas que ainda não haviam sido estudados anteriormente. Entre os dados fornecidos foram custo diretos e indiretos, tempo médio de produção por categoria, entre outros dados. Com o objetivo de identificar possíveis gargalos de produção que é responsável pela etapa mais lenta no processo produtivo (Leão, 2019).

O estudo se limita ao setor produtivo, os dados coletados foram apurados e apresentados na forma descritiva, em tabelas e quadros. Inicialmente foi calculado o tempo médio de produção de cada produto, junto com seus relativos custos. A partir desse ponto, foi então calculada a capacidade de produção de cada setor, identificando os pontos a serem melhorados.

\section{RESULTADOS}

Os departamentos da indústria estudada são divididos em três, cada um é uma etapa no processo produtivo, para melhor ilustrar, foi desenhado um mapa baixo da indústria, representado pela Figura 1, na parte de anexos (Folha 21). A análise do layout físico e a estrutura da empresa faz parte do processo de análise da capacidade produtiva, constata-se a forma como é alocado cada recurso durante as operações (Boff, 2013).

Observa-se na Figura 1, em amarelo representa 0 primeiro departamento/etapa, intitulado como "Setor de Corte", é a parte do processo onde são realizados os cortes dos tecidos para a produção dos produtos. Dentro desse setor possui uma mesa de talharia/enfesto de 6 metros por 1,60 metro, máquinas de corte de tecido, entre outros equipamentos para auxiliarem no setor.

Esse departamento é operado por dois colaboradores, sua produção é relacionada com a demanda de novos pedidos, dentro dos produtos cortados no setor, estão em estudo 5 tamanhos de capas de máquina, 4 tamanhos de protetor, 11 tamanhos de forro para passar roupa e 2 tamanhos de almofada para passar 
roupa, totalizando 22 produtos cortados de maneira diferente, levando em consideração o tempo médio de corte e o custo do tecido cortado. As capas de máquina possuem variação de cor, sendo que as cores não alteram nos custos de produção, elas passam pelo mesmo processo e possuem os mesmos custos, apenas alterando o tecido utilizado, não tendo a necessidade de diferenciar os dados apresentado por cor.

Os custos que englobam o departamento de corte, são custos de matériaprima e o custo de mão de obra direta (MOD), cada categoria de produto tem um custo diferente MOD, baseado no tempo médio levado para esticar, de medir e a quantidade máxima que pode ser cortada por vez, um exemplo são as capas de máquinas, 2 unidades por folha, podendo ser cortados máximo 50 folhas por vez, diferente do forro para passar roupa Ref. 0144, que pode ser cortado 20 unidades por folha de tecido.

O Quadro 02 ilustra a quantidade produzida por tempo do departamento, junto com o tempo médio gasta para cortar, esticar e preparar o tecido. $O$ primeiro passo dos colaboradores do setor de corte é definir a quantidade de produtos que devem ser cortados na chegada de novos pedidos, deduzindo os produtos em estoque, estipulando a quantidade necessária pela diferença. Para cada categoria de produto foi calculado o tempo médio utilizado para preparar, esticar e cortar o tecido, junto com o número máximo de folhas que podem ser cortadas de uma única fez e a quantidade máxima de unidades por folha.

Para critério de rateio o primeiro departamento utiliza o tempo médio por corte em relação as unidades por ele produzidas, para ratear o custo de MOD do setor de corte. Após os colaboradores terminarem os cortes, eles são remanejados para o setor de embalagem e acabamento. 


\begin{tabular}{|c|c|c|c|c|c|c|c|c|c|}
\hline Categoria & Produto & Ref. & Preparação & $\begin{array}{c}\text { Tempo } \\
\text { Média } \\
\text { por Folha }\end{array}$ & $\begin{array}{c}\text { Marcação e } \\
\text { Corte }\end{array}$ & $\begin{array}{l}\text { Quantida } \\
\text { por Folha }\end{array}$ & $\begin{array}{c}\text { Máximo } \\
\text { de Folhas }\end{array}$ & $\begin{array}{c}\text { Tempo Médio } \\
\text { por Corte }\end{array}$ & $\begin{array}{c}\text { Unidades } \\
\text { por } \\
\text { Corte }\end{array}$ \\
\hline \multirow{5}{*}{$\begin{array}{c}\text { Capa Para } \\
\text { Lavadora e } \\
\text { Secadora de } \\
\text { Roupas }\end{array}$} & $\mathrm{P}$ & 0045 & 00:04:00 & 00:00:45 & $00: 20: 00$ & 2 & 50 & 01:01:30 & 100 \\
\hline & M & 0052 & 00:04:00 & 00:00:45 & 00:20:00 & 2 & 50 & 01:01:30 & 100 \\
\hline & G & 0069 & 00:04:00 & $00: 00: 45$ & $00: 20: 00$ & 2 & 50 & 01:01:30 & 100 \\
\hline & GG & 0076 & 00:04:00 & $00: 00: 45$ & 00:25:00 & 2 & 50 & 01:06:30 & 100 \\
\hline & TU & 0083 & 00:04:00 & 00:00:45 & 00:25:00 & 2 & 50 & 01:06:30 & 100 \\
\hline \multirow{8}{*}{$\begin{array}{c}\text { Forro para tábua } \\
\text { de passar roupas } \\
\text { com espuma }\end{array}$} & $0,35 \times 1,10 m-n=1$ & 0144 & $00: 04: 00$ & 00:01:00 & 00:38:00 & 20 & 40 & 01:22:00 & 800 \\
\hline & $0,38 \times 1,35 m-n=2$ & 0151 & 00:04:00 & 00:01:00 & $00: 32: 00$ & 15 & 40 & 01:16:00 & 600 \\
\hline & $0,46 \times 1,10 m-n \circ 3$ & 0169 & 00:04:00 & 00:01:00 & $00: 30: 00$ & 15 & 40 & 01:14:00 & 600 \\
\hline & $0,50 \times 1,10 m-n=4$ & 0175 & 00:04:00 & 00:01:00 & 00:25:00 & 10 & 40 & 01:09:00 & 400 \\
\hline & $0,57 \times 1,35 m-n=5$ & 0229 & 00:04:00 & 00:01:00 & $00: 30: 00$ & 10 & 40 & 01:14:00 & 400 \\
\hline & $0,35 \times 0,75 m-n=6$ & 0854 & 00:04:00 & 00:01:00 & $00: 25: 00$ & 32 & 40 & 01:09:00 & 1280 \\
\hline & $0,46 \times 1,20 m-n=7$ & 0861 & 00:04:00 & 00:01:00 & $00: 30: 00$ & 12 & 40 & 01:14:00 & 480 \\
\hline & $0,57 \times 1,45 m-n=8$ & 0878 & 00:04:00 & 00:01:00 & 00:25:00 & 10 & 40 & 01:09:00 & 400 \\
\hline \multirow{4}{*}{$\begin{array}{c}\text { Protetor de } \\
\text { Roupas }\end{array}$} & $48 \mathrm{~cm} \times 60 \mathrm{~cm}$ & 0236 & 00:04:00 & 00:01:15 & $00: 35: 00$ & 12 & 80 & 02:19:00 & 960 \\
\hline & $36 \mathrm{~cm} \times 50 \mathrm{~cm}$ & 0243 & 00:04:00 & 00:01:15 & 00:35:00 & 20 & 80 & 02:19:00 & 1600 \\
\hline & $22 \mathrm{~cm} \times 30 \mathrm{~cm}$ & 0250 & 00:04:00 & 00:01:15 & 00:39:00 & 48 & 80 & 02:23:00 & 3840 \\
\hline & $30 \mathrm{~cm} \times 1 \mathrm{~m}$ & 0311 & 00:04:00 & $00: 01: 15$ & $00: 32: 00$ & 8 & 80 & 02:16:00 & 640 \\
\hline \multirow{2}{*}{$\begin{array}{l}\text { Almofada Para } \\
\text { passar roupa }\end{array}$} & Almofada Mesa & 0014 & $00: 04: 00$ & $00: 01: 40$ & $00: 25: 00$ & 15 & 24 & 01:09:00 & 360 \\
\hline & Almofada Tábua & 0021 & 00:04:00 & 00:01:40 & 00:30:00 & 20 & 24 & 01:14:00 & 480 \\
\hline
\end{tabular}

\section{Quadro 02. Tempo x Quantidade por corte.}

Fonte: Dados da Pesquisa.

No Quadro 02, as divisões, Ref. são os últimos 4 dígitos do código EAN, é a referência para o cadastro do produto. Preparação é um tempo médio levado para colocar os tecidos na mesa de enfesto, fazer as medições iniciais. Tempo médio por folha é um tempo médio que os colaboradores levam para esticar uma folha de tecido. Marcação e Corte é um tempo médio levado para os colaboradores marcarem o tecido e cortá-lo. Quantidade por Folha é a quantidade de produtos por folha. Máximo de Folhas é a quantidade máxima de folhas que a máquina de corte consegue cortar por vez. Tempo Médio por Corte é o tempo total levado para cortar. Unidades por Corte é o máximo que pode ser cortado por vez de unidades. O tempo é evidenciado na tabela pela ordem de hora, minuto, segundo.

O departamento de corte, usando como um dia de trabalho de 9 horas, pode ter a capacidade produtiva estimada por produto, representado pelo Quadro 03. 


\begin{tabular}{|c|c|c|c|c|}
\hline \multicolumn{5}{|c|}{ CAPACIDADE MÉDIA DE PRODUÇÃO POR DIA NO DEPARTAMENTO CORTE } \\
\hline Categoria & Produto & Ref. & Tempo & $\begin{array}{l}\text { Quantidade total } \\
\text { média por dia }\end{array}$ \\
\hline \multirow{5}{*}{$\begin{array}{c}\text { Capa para } \\
\text { Lavadora e } \\
\text { Secadora de } \\
\text { Roupas } \\
\end{array}$} & $\mathrm{P}$ & 0045 & 09:00:00 & 878 \\
\hline & $\mathrm{M}$ & 0052 & 09:00:00 & 878 \\
\hline & $\mathrm{G}$ & 0069 & 09:00:00 & 878 \\
\hline & GG & \begin{tabular}{|l|}
0076 \\
\end{tabular} & 09:00:00 & 812 \\
\hline & TU & \begin{tabular}{|l|}
0083 \\
\end{tabular} & 09:00:00 & 812 \\
\hline \multirow{8}{*}{$\begin{array}{c}\text { Forro para Tábua } \\
\text { de Passar } \\
\text { Roupas }\end{array}$} & $0,35 \times 1,10 m-n=1$ & 0144 & 09:00:00 & 5.268 \\
\hline & $0,38 \times 1,35 m-n=2$ & \begin{tabular}{|l|}
0151 \\
\end{tabular} & 09:00:00 & 4.263 \\
\hline & $0,46 \times 1,10 m-n=3$ & \begin{tabular}{|l|}
0169 \\
\end{tabular} & 09:00:00 & 4.378 \\
\hline & $0,50 \times 1,10 m-n=4$ & \begin{tabular}{|l|}
0175 \\
\end{tabular} & 09:00:00 & 3.130 \\
\hline & $0,57 \times 1,35 m-n=5$ & \begin{tabular}{|l|}
0229 \\
\end{tabular} & 09:00:00 & 2.919 \\
\hline & $0,35 \times 0,75 m-n=6$ & \begin{tabular}{|l|}
0854 \\
\end{tabular} & 09:00:00 & 10.017 \\
\hline & $0,46 \times 1,20 m-n=7$ & \begin{tabular}{|l|}
0861 \\
\end{tabular} & 09:00:00 & 3.503 \\
\hline & $0,57 \times 1,45 m-n=8$ & \begin{tabular}{|l|}
0878 \\
\end{tabular} & 09:00:00 & 3.130 \\
\hline \multirow{4}{*}{$\begin{array}{c}\text { Protetor de } \\
\text { Roupas }\end{array}$} & $48 \mathrm{~cm} \times 60 \mathrm{~cm}$ & 0236 & 09:00:00 & 3.729 \\
\hline & $36 \mathrm{~cm} \times 50 \mathrm{~cm}$ & 0243 & 09:00:00 & 6.216 \\
\hline & $22 \mathrm{~cm} \times 30 \mathrm{~cm}$ & 0250 & 09:00:00 & 14.501 \\
\hline & $30 \mathrm{~cm} \times 1 \mathrm{~m}$ & 0311 & 09:00:00 & 2.541 \\
\hline \multirow{2}{*}{$\begin{array}{l}\text { Almofada para } \\
\text { Passar Roupa }\end{array}$} & Almofada Mesa & \begin{tabular}{|l|}
0014 \\
\end{tabular} & 09:00:00 & 2.817 \\
\hline & Almofada Tábua & \begin{tabular}{|l|}
0021 \\
\end{tabular} & 09:00:00 & 3.503 \\
\hline
\end{tabular}

Quadro 3. Capacidade produtiva por dia.

Fonte: Dados da Pesquisa.

A segunda etapa no processo é representada pelo departamento de costura, ilustrado na cor verde, anexo, Figura 1. Esse setor possui 6 máquinas de costura, entre elas são 4 retas e 2 overloques, as máquinas foram substituídas por modelos mais novos nos últimos anos, aumentando a produtividade e agilizando os processos anteriormente manuais. O setor dispõe de 4 costureiras e 2 auxiliares. Após o término dos cortes de tecido, os colaboradores do departamento são remanejados para o departamento de costura, onde passa a ter 4 auxiliares.

Todas as categorias analisadas, excluindo forro para tábua, são direcionadas para a costura após o corte, onde recebem os primeiros tratamentos. Um exemplo são as capas de máquinas, primeiramente são costuradas pelo overloque, onde são juntas suas partes, posteriormente vão para as máquinas retas onde obtém o acabamento, elástico e viés, após esse processo elas estão prontas para irem ao setor de embalagem. Os forros para tábua antes de irem para a costura necessitam receber a espuma, colocada manualmente em cada forro pelos auxiliares. 
Para melhor entendimento desses processos produtivos é descrito as etapas do departamento de costura de cada item em análise.

A capa de máquina é composta de 2 partes, a saia e a tampa, elas são usadas como proteção das máquinas de lavar roupa, principalmente as que têm exposição ao tempo ou são mantidas em locais externos.

A primeira etapa do processo se inicia com a saia, ela é composta de 3 partes iguais, as laterais e a parte frontal, são costuras no overloque, de maneira a formar uma proteção na parte frontal e lateral da máquina.

A tampa é a parte superior, que é utilizada para as máquinas com abertura superior, ela é composta de 3 partes, 2 laterais pequenas em forma de trapézio e uma superior em forma de quadrado, são costuradas juntas pelo overloque e ao término desse processo as capas vão para a costura reta.

A segunda etapa consiste no acabamento, feito pela máquina reta, onde é costurado um viés por toda borda da capa, junto com uma etiqueta e um elástico na parte de trás da saia, para melhor fixação na máquina, após esse processo a capa é encaminha para o setor de embalagem.

Já o forro para tábua é confeccionado através de um tecido metalizado que auxilia no isolamento térmico e para refletir o calor, junto com uma espuma para melhorar a aderência do ferro de passar roupa com a tábua. Usado para substituir o forro da tábua de passar roupa quando apresenta desgaste, rasgos, manchas ou produzido com material inferior.

Seguem algumas etapas:

$\left.1^{a}\right)$ Ao término do corte, o tecido é sobreposto sobre uma folha de espuma já cortada do tamanho do tecido, colocado de forma manual pelos auxiliares;

$2^{a}$ ) Se inicia pela máquina reta, onde é costurado um viés em volta do forro, nas partes laterais junto com o viés é costurado 4 pedaços de cadarço, 2 em cada lado, com a funcionalidade de fixação/amarração na tábua, na parte do bico, onde forma uma semi elipse, é posto junto com o viés um cordão para regulagem do bico na tábua;

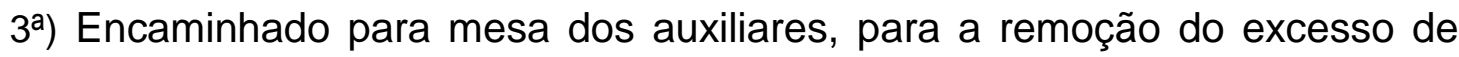
linhas e de espuma que eventualmente pode sobrar na cabeceira do forro; 
4ㄹ) O fechamento da cabeceira é feito na máquina reta, onde é costurado um viés junto com uma etiqueta, ao finalizar esse processo os auxiliares encaminham os forros para o setor de embalagem.

O protetor de roupa, também conhecido mais popularmente como saquinho de lavar, é utilizado para lavagem de todos os tipos roupas, com o intuito de diminuir o desgaste gerado pela máquina, gerando na hora de lavar, podendo lavar roupas de cores e tipos diferentes na mesma máquina, sem se preocupar com penugem e o atrito entre as roupas. A produção do protetor é iniciada pela máquina reta, onde é costurado o zíper, o elástico de proteção do zíper e a etiqueta. Sendo que essa etapa é feita pelo overloque, onde é costurado as laterais do protetor, ao finalizar esse processo os protetores são encaminhados para o setor de embalagem. A almofada para passar roupa, é uma criação patenteada pela Alfa, o primeiro produto a ser fabricado pela empresa. É uma alternativa para substituir a tábua de passar roupa, uma almofada térmica, que reflete o calor do ferro de passar roupa, passando-a dos dois lados ao mesmo tempo, diminuindo o tempo levado para passar e consequentemente economizando energia. Possui propriedades isolantes, podendo ser usada em qualquer superfície como mogno, vidro, mármore, madeira ou até mesmo na cama e no sofá, não transferindo o calor gerado pelo ferro de passar diretamente para a superfície.

$1^{\text {a }}$ etapa: a almofada possui dois tecidos, um metalizado e um antiderrapante de poliuretano, são costurados pela máquina reta com um viés, a parte lateral e a superior, deixando a parte da cabeceira aberta para posteriormente receber a espuma;

$2^{\text {a }}$ etapa: a colocação de uma espuma especial com isolamento térmico, pelos auxiliares;

$3^{\text {a }}$ etapa: o fechamento da cabeceira, com o viés e a colocação da etiqueta, após o término da costura, a almofada é encaminha para o setor de embalagem.

O critério de avaliação dos custos é baseado no tempo médio de produção por unidade, descontado o tempo médio de remanejo dos auxiliares do setor de costura para embalagem, que é próximo dos $20 \%$. No Quadro 04, é abordado o tempo médio por unidade no Departamento de Costura. 


\begin{tabular}{|c|c|c|c|c|c|c|c|}
\hline Categoria & Produto & Ref. & 10 & 20 & 3o & 40 & $\begin{array}{c}\text { Tempo Médio } \\
\text { por unidade }\end{array}$ \\
\hline \multirow{5}{*}{$\begin{array}{c}\text { Capa Para Lavadora e } \\
\text { Secadora de Roupas }\end{array}$} & $\mathrm{P}$ & 0045 & 00:01:30 & 00:04:50 & & & 00:06:20 \\
\hline & $\mathrm{M}$ & 0052 & 00:01:53 & 00:05:10 & & & 00:07:03 \\
\hline & G & 0069 & 00:02:06 & 00:05:12 & & & 00:07:18 \\
\hline & GG & 0076 & $00: 02: 20$ & $00: 05: 37$ & & & 00:07:57 \\
\hline & TU & 0083 & 00:02:30 & 00:06:12 & & & 00:08:42 \\
\hline \multirow{8}{*}{$\begin{array}{c}\text { Forro para tábua de } \\
\text { passar roupas com } \\
\text { espuma }\end{array}$} & $0,35 \times 1,10 m-n=1$ & 0144 & $00: 00: 10$ & $00: 01: 56$ & $00: 00: 14$ & $00: 00: 28$ & $00: 02: 48$ \\
\hline & $0,38 \times 1,35 m-n=2$ & 0151 & $00: 00: 11$ & $00: 02: 07$ & $00: 00: 15$ & $00: 00: 29$ & 00:03:02 \\
\hline & $0,46 \times 1,10 m-n=3$ & 0169 & $00: 00: 12$ & 00:02:10 & 00:00:19 & 00:00:30 & 00:03:11 \\
\hline & $0,50 \times 1,10 m-n \circ 4$ & 0175 & 00:00:09 & 00:02:08 & 00:00:18 & 00:00:30 & 00:03:05 \\
\hline & $0,57 \times 1,35 m-n=5$ & 0229 & 00:00:18 & $00: 02: 45$ & 00:00:19 & $00: 00: 32$ & 00:03:54 \\
\hline & $0,35 \times 0,75 m-n=6$ & 0854 & 00:00:08 & $00: 01: 28$ & $00: 00: 10$ & $00: 00: 27$ & $00: 02: 13$ \\
\hline & $0,46 \times 1,20 m-n=7$ & 0861 & $00: 00: 15$ & $00: 02: 12$ & $00: 00: 17$ & 00:00:30 & 00:03:14 \\
\hline & $0,57 \times 1,45 m-n \circ 8$ & 0878 & 00:00:16 & 00:03:27 & 00:00:19 & 00:00:31 & 00:04:33 \\
\hline \multirow{4}{*}{ Protetor de Roupas } & $48 \mathrm{~cm} \times 60 \mathrm{~cm}$ & 0236 & $00: 01: 40$ & $00: 00: 40$ & & & $00: 02: 20$ \\
\hline & $36 \mathrm{~cm} \times 50 \mathrm{~cm}$ & 0243 & 00:01:30 & 00:00:35 & & & 00:02:05 \\
\hline & $22 \mathrm{~cm} \times 30 \mathrm{~cm}$ & 0250 & 00:01:00 & 00:00:25 & & & 00:01:25 \\
\hline & $30 \mathrm{~cm} \times 1 \mathrm{~m}$ & 0311 & 00:01:03 & 00:00:40 & & & 00:01:43 \\
\hline \multirow{2}{*}{$\begin{array}{c}\text { Almofada Para passar } \\
\text { roupa }\end{array}$} & Almofada Mesa & 0014 & $00: 00: 45$ & $00: 00: 30$ & $00: 00: 20$ & & $00: 01: 35$ \\
\hline & Almofada Tábua & 0021 & $00: 00: 42$ & 00:00:35 & $00: 00: 26$ & & 00:01:43 \\
\hline
\end{tabular}

\section{Quadro 4. Tempo médio por unidade Departamento de costura}

Fonte: Dados Primários.

A segunda etapa é a parte mais lenta do processo produtivo, criando um gargalo de produção na parte da costura, em períodos de grande demanda de produção, um dos auxiliares é remanejado para a máquina e costura, agilizando alguns possíveis gargalos do processo. Esse setor usa um dia de trabalho de 9 horas que pode ter a capacidade produtiva estimada por produto, representado no Quadro 05, usando como base o trabalho de quatro costureiras. 
Capacidade Produtiva e Análise De Custo Em Uma Empresa No Setor De Confecção João Paulo Pereira, Marcelo Antonio Pierri Junior

\begin{tabular}{|c|c|c|c|c|}
\hline Categoria & Produto & Ref. & Tempo & $\begin{array}{l}\text { Quantidade total } \\
\text { média por dia }\end{array}$ \\
\hline \multirow{5}{*}{$\begin{array}{c}\text { Capa para } \\
\text { Lavadora e } \\
\text { Secadora de } \\
\text { Roupas } \\
\end{array}$} & $\mathrm{P}$ & 0045 & 09:00:00 & 341 \\
\hline & $\mathrm{M}$ & 0052 & 09:00:00 & 306 \\
\hline & $\mathrm{G}$ & 0069 & 09:00:00 & 296 \\
\hline & GG & 0076 & 09:00:00 & 272 \\
\hline & TU & 0083 & 09:00:00 & 248 \\
\hline \multirow{8}{*}{$\begin{array}{c}\text { Forro para Tábua } \\
\text { de Passar } \\
\text { Roupas }\end{array}$} & $0,35 \times 1,10 m-n=1$ & 0144 & 09:00:00 & 771 \\
\hline & $0,38 \times 1,35 m-n=2$ & 0151 & 09:00:00 & 712 \\
\hline & $0,46 \times 1,10 m-n=3$ & 0169 & 09:00:00 & 679 \\
\hline & $0,50 \times 1,10 m-n=4$ & 0175 & 09:00:00 & 701 \\
\hline & $0,57 \times 1,35 m-n=5$ & 0229 & 09:00:00 & 554 \\
\hline & $0,35 \times 0,75 m-n=6$ & 0854 & 09:00:00 & 974 \\
\hline & $0,46 \times 1,20 m-n=7$ & 0861 & 09:00:00 & 668 \\
\hline & $0,57 \times 1,45 m-n=8$ & 0878 & 09:00:00 & 475 \\
\hline \multirow{4}{*}{$\begin{array}{c}\text { Protetor de } \\
\text { Roupas }\end{array}$} & $48 \mathrm{~cm} \times 60 \mathrm{~cm}$ & 0236 & 09:00:00 & 926 \\
\hline & $36 \mathrm{~cm} \times 50 \mathrm{~cm}$ & 0243 & 09:00:00 & 1.037 \\
\hline & $22 \mathrm{~cm} \times 30 \mathrm{~cm}$ & 0250 & 09:00:00 & 1.525 \\
\hline & $30 \mathrm{~cm} \times 1 \mathrm{~m}$ & 0311 & 09:00:00 & 1.258 \\
\hline \multirow{2}{*}{$\begin{array}{c}\text { Almofada para } \\
\text { Passar Roupa }\end{array}$} & Almofada Mesa & 0014 & 09:00:00 & 1.364 \\
\hline & Almofada Tábua & 0021 & 09:00:00 & 1.258 \\
\hline
\end{tabular}

Quadro 05. Tempo médio por unidade Departamento de costura

Fonte: Dados Primários.

A partir das informações do Quadro 05, uma estimativa média de produção por departamento e categoria ao dia; as capas de máquina, 852 unidades no setor de corte e 293 unidades no de costura; os forros, 4.576 unidades no setor de corte e 692 unidades no de costura; os protetores, 6.747 unidades no setor de corte e 1.186 unidades no de costura; e as almofadas, 3.160 unidades no setor de corte e 1.311 unidades no de costura.

A terceira etapa do processo produtivo é representada pelo departamento de embalagem, ilustrado em azul na Figura 1, na parte de anexos. Esse setor é responsável pelo controle de qualidade, empacotar/embalar os produtos, separar os pedidos para expedição e o controle dos estoques.

Esse departamento utiliza os mesmos colaboradores do setor de corte e os de costura, que são remanejados conforme a necessidade e a demanda de produção, a sua operação não é linear e nem contínua, utiliza critérios diferenciados para avaliar os custos, baseado no tempo total médio levado para a finalização dos pedidos auferindo uma porcentagem de tempo utilizado pelos colaboradores. Sendo 
$20 \%$ do custo total de MOD do departamento de costura e $15 \%$ do departamento de corte.

Os custos são representados por critérios de rateios diferentes, no departamento de corte é rateado pela hora de mão de obra direta, com base no tempo médio de produção por unidade, considerando o tempo médio mensal usufruído pelos colaboradores de $37 \%$ no departamento de corte, descontado o tempo de remanejamento em outros departamentos, $48 \%$ no setor de costura e $15 \%$ no setor de embalagem, usando a base de cálculo, ((MOD * 37\%) / Tempo total de produção * Tempo por Unidade), para atingir o valor de custo indireto por unidade. No departamento de costura o critério é o tempo médio de produção por unidade, com base na MOD das costureiras, é o departamento com o maior custo de mão de obra, devido a ser a etapa do processo mais extensa.

Os produtos terminados pela costura são encaminhados ao departamento de embalagem, onde todos são examinados com o intuito de verificar possíveis defeitos de fabricação, como rasgos, manchas, partes descosturadas, entre outros. Os que forem reprovados pelo teste de qualidade, são reparados ou descartados, dependendo o seu defeito. Os que passam, são dobrados e empacotados em suas respectivas embalagens, exceto as almofadas que são embaladas por inteiro, sem serem dobradas. Os produtos, após serem embalados, são postos em sacos de 6 unidades e os protetores para roupa em sacos de 12 unidades, em seguida encaminhados para o depósito ou para expedição.

O departamento de embalagem trabalha em correlação ao departamento de costura, por ser uma parte ágil do processo, apenas um colaborar consegue acompanhar o ritmo de produção de 3 máquinas de costura, variando o produto produzido, caso as quantidades comecem a se acumular, o setor de costurar remaneja mais um auxiliar para o setor de embalagem. Dando um exemplo mais prático, enquanto as costureiras estão produzindo a $2^{\text {a }}$ etapa do forro ou da capa, os auxiliares são remanejados para o setor de embalagem, já que é uma etapa do processo que não se tem tanta necessidade de muitos auxiliares na costura, assim usando o tempo para fazer os processos indiretos de produção. Sua produção é superior a quantidade produzida pela costura, tornando a sua capacidade produtiva a mesma que o departamento de costura. 


\begin{tabular}{|c|c|c|}
\hline \multirow{2}{*}{ Categoria } & Produto & Ref. \\
\hline \multirow{2}{*}{$\begin{array}{c}\text { Capa Para } \\
\text { Lavadora e }\end{array}$} & $\mathrm{P}$ & 0045 \\
\cline { 2 - 3 } $\begin{array}{c}\text { Secadora de } \\
\text { Roupas }\end{array}$ & $\mathrm{G}$ & 0052 \\
\cline { 2 - 3 } & $\mathrm{GG}$ & 0069 \\
\hline
\end{tabular}

\begin{tabular}{|c|c|c|c|c|c|c|c|c|c|c|}
\hline \multicolumn{11}{|c|}{ Departamentos } \\
\hline \multicolumn{2}{|c|}{ Corte } & \multicolumn{9}{|c|}{ Costura } \\
\hline Tecido & $\begin{array}{l}\text { MOD por } \\
\text { Unidade }\end{array}$ & Viés & Elastico & Linha & Cadarço & Ziper & Espuma & Etiqueta & Cordão & MOD \\
\hline $\mathrm{R} \$ 5,81$ & $\mathrm{R} \$ \quad 1,14$ & $\mathrm{R} \$ \mathbf{2 , 0 2}$ & $\mathrm{R} \$ 0,18$ & $\mathrm{R} \$ 0,23$ & & & & $R \$ 0,02$ & & $R \$ 4,26$ \\
\hline $\mathrm{R} \$ 5,92$ & $\mathrm{R} \$ \quad 1,14$ & $R \$ 2,05$ & $\mathrm{R} \$ 0,18$ & $R \$ 0,23$ & & & & $R \$ 0,02$ & & $\mathrm{R} \$ 4,74$ \\
\hline$R \$ 5,98$ & $\mathrm{R} \$ \quad 1,14$ & $\mathrm{R} \$ 2,10$ & $\mathrm{R} \$ 0,18$ & $R \$ 0,23$ & & & & $R \$ 0,02$ & & $\mathrm{R} \$ 4,91$ \\
\hline $\mathrm{R} \$ 6,70$ & $\mathrm{R} \$ 1,24$ & $\mathrm{R} \$ 2,27$ & $\mathrm{R} \$ 0,18$ & $\mathrm{R} \$ 0,23$ & & & & $R \$ 0,02$ & & $\mathrm{R} \$ 5,35$ \\
\hline$R \$ 5,83$ & $\mathrm{R} \$ \quad 1,24$ & $\mathrm{R} \$ 1,81$ & & $R \$ 0,23$ & & & & $R \$ 0,02$ & & $\mathrm{R} \$ 5,85$ \\
\hline
\end{tabular}

\begin{tabular}{|c|l|l|}
\hline \multirow{4}{*}{$\begin{array}{c}\text { Forro para } \\
\text { tábua de }\end{array}$} & $0,35 \times 1,10 m-n=1$ & 0144 \\
\cline { 2 - 3 } passar roupas & $0,38 \times 1,35 m-n=2$ & 0151 \\
\cline { 2 - 3 } com espuma & $0,46 \times 1,10 m-n=3$ & 0169 \\
\cline { 2 - 3 } & $0,50 \times 1,10 m-n=4$ & 0175 \\
\cline { 2 - 3 } & $0,57 \times 1,35 m-n=5$ & 0229 \\
\cline { 2 - 3 } & $0,35 \times 0,75 m-n=6$ & 0854 \\
\cline { 2 - 3 } & $0,47 \times 1,20 m-n=7$ & 0861 \\
\hline
\end{tabular}

\begin{tabular}{|l|ll|}
\hline$R \$ 1,88$ & $R \$$ & 0,19 \\
\hline$R \$ 1,69$ & $R \$$ & 0,24
\end{tabular} $R \$ 2,69 \quad \mathrm{R} \$ \quad 0,24$ \begin{tabular}{|l|ll|}
\hline$R \$ 2,51$ & $R \$$ & 0,23 \\
\hline$R \$ 2,83$ & $R \$$ & 0,32 \\
\hline
\end{tabular} $R \$ 2,83 \quad R \$ \quad 0,32$ $R \$ 3,84$ R\$ 0,34 \begin{tabular}{|l|ll|}
\hline$R \$ 1,26$ & $R \$$ & 0,10 \\
\hline
\end{tabular} $R \$ 2,74 \quad R \$ \quad 0,29$ \begin{tabular}{|l|ll|}
\hline$R \$ 3,70$ & $R \$ 1$ & 0,32 \\
\hline
\end{tabular}

\begin{tabular}{|c|c|c|}
\hline \multirow{3}{*}{$\begin{array}{c}\text { Protetor de } \\
\text { Roupas }\end{array}$} & $48 \mathrm{~cm} \times 60 \mathrm{~cm}$ & 0236 \\
\cline { 2 - 3 } & $36 \mathrm{~cm} \times 50 \mathrm{~cm}$ & 0243 \\
\cline { 2 - 3 } & $22 \mathrm{~cm} \times 30 \mathrm{~cm}$ & 0250 \\
\cline { 2 - 3 } & $30 \mathrm{~cm} \times 1 \mathrm{~m}$ & 0311 \\
\hline
\end{tabular}

\begin{tabular}{|l|ll|}
\hline$R \$ 1,47$ & $R \$$ & 0,27 \\
\hline
\end{tabular} \begin{tabular}{llll}
\hline$\$$ & 0,93 & $R \$$ & 0,16 \\
\hline
\end{tabular} \begin{tabular}{|l|ll|}
\hline$R \$ 0,39$ & $R \$$ & 0,07 \\
\hline$R \$ 1,51$ & $R \$$ & 0,40
\end{tabular} \begin{tabular}{|l|ll|}
\hline$R \$ 1,51$ & $R \$$ & 0,40 \\
\hline
\end{tabular}

\begin{tabular}{|c|c|c|c|c|c|c|c|}
\hline $\mathrm{R} \$ 0,60$ & $\mathrm{R} \$ 0,16$ & $\mathrm{R} \$ 0,08$ & $\mathrm{R} \$$ & 0,75 & $\mathrm{R} \$ 0,02$ & $\mathrm{R} \$ 0,03$ & $\mathrm{R} \$ 1,88$ \\
\hline $\mathrm{R} \$ \mathbf{0 , 7 3}$ & $\mathrm{R} \$ 0,16$ & $\mathrm{R} \$ 0,08$ & $\mathrm{R} \$$ & 1,03 & $R \$ 0,02$ & $\mathrm{R} \$ 0,03$ & $\mathrm{R} \$ 2,04$ \\
\hline $\mathrm{R} \$ 0,63$ & $\mathrm{R} \$ 0,16$ & $\mathrm{R} \$ 0,08$ & $\mathrm{R} \$$ & 0,98 & $\mathrm{R} \$ 0,02$ & $\mathrm{R} \$ 0,03$ & $\mathrm{R} \$ 2,14$ \\
\hline $\mathrm{R} \$ \mathbf{0 , 6 4}$ & $\mathrm{R} \$ 0,16$ & $\mathrm{R} \$ 0,08$ & $\mathrm{R} \$$ & 1,06 & $R \$ 0,02$ & $R \$ 0,03$ & $\mathrm{R} \$ 2,07$ \\
\hline $\mathrm{R} \$ 0,77$ & $\mathrm{R} \$ 0,16$ & $\mathrm{R} \$ 0,08$ & $\mathrm{R} \$$ & 1,61 & $\mathrm{R} \$ 0,02$ & $\mathrm{R} \$ 0,03$ & $\mathrm{R} \$ 2,62$ \\
\hline $\mathrm{R} \$ \mathbf{0 , 4 4}$ & $\mathrm{R} \$ 0,16$ & $\mathrm{R} \$ \mathbf{0 , 0 8}$ & $\mathrm{R} \$$ & 0,52 & $\mathrm{R} \$ 0,02$ & $R \$ 0,03$ & $\mathrm{R} \$ 1,49$ \\
\hline $\mathrm{R} \$ 0,67$ & $\mathrm{R} \$ 0,16$ & $\mathrm{R} \$ 0,08$ & $\mathrm{R} \$$ & 1,07 & $R \$ 0,02$ & $\mathrm{R} \$ 0,03$ & $\mathrm{R} \$ 2,18$ \\
\hline $\mathrm{R} \$ 0,82$ & $\mathrm{R} \$ 0,16$ & $\mathrm{R} \$ 0,08$ & $\mathrm{R} \$$ & 1,51 & $R \$ 0,02$ & $R \$ 0,03$ & $\mathrm{R} \$ 3,06$ \\
\hline
\end{tabular}

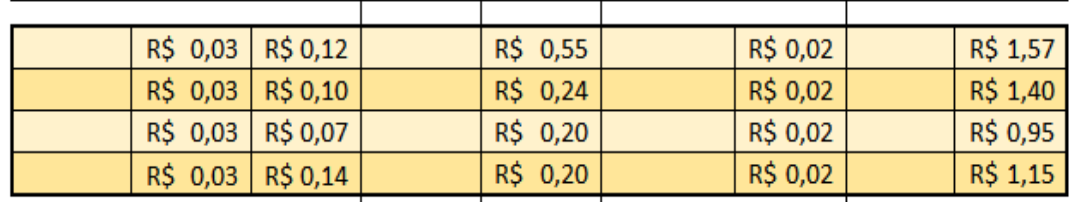

\begin{tabular}{|l|l|l|}
\hline Almofada & Almofada Mesa & 0014 \\
\hline
\end{tabular}

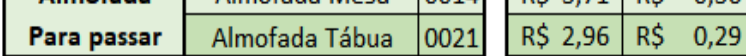

\begin{tabular}{|c|c|c|c|c|c|}
\hline $\mathrm{R} \$ 0$ & 18 & & 3,24 & 0,02 & $\mathrm{R} \$ 1, \mathrm{C}$ \\
\hline$R \$ 0,71$ & $\mathrm{R} \$ 0,18$ & $\mathrm{R} \$ 0,08$ & $\mathrm{R} \$ \mathbf{2 , 6 4}$ & $R \$ 0,02$ & $\mathrm{R} \$ 1,15$ \\
\hline
\end{tabular}

\begin{tabular}{|c|c|c|c|c|}
\hline \multicolumn{5}{|c|}{ Embalagem } \\
\hline \multicolumn{2}{|c|}{ Embalagem } & $\begin{array}{l}\text { Etiquetas } \\
\text { Adesiva }\end{array}$ & $\begin{array}{c}\text { Mat. } \\
\text { Consumo }\end{array}$ & MOD \\
\hline $\mathrm{R} \$$ & 0,16 & $\mathrm{R} \$ 0,02$ & $\mathrm{R} \$ 0,08$ & $\mathrm{R} \$ 0,50$ \\
\hline $\mathrm{R} \$$ & 0,16 & $R \$ 0,02$ & $\mathrm{R} \$ 0,08$ & $\mathrm{R} \$ 0,55$ \\
\hline $\mathrm{R} \$$ & 0,16 & $\mathrm{R} \$ 0,02$ & $\mathrm{R} \$ 0,08$ & $\mathrm{R} \$ 0,57$ \\
\hline $\mathrm{R} \$$ & 0,16 & $\mathrm{R} \$ 0,02$ & $\mathrm{R} \$ \mathbf{0 , 0 8}$ & $\mathrm{R} \$ 0,62$ \\
\hline $\mathrm{RS}$ & 0,16 & $\mathrm{R} \$ 0,02$ & $\mathrm{R} \$ 0,08$ & $\mathrm{R} \$ 0,67$ \\
\hline
\end{tabular}

\begin{tabular}{|ll|l|l|l|l|}
\hline$R \$$ & 0,18 & $R \$ 0,02$ & $R \$ 0,06$ & $R \$ 0,42$ \\
\hline
\end{tabular} \begin{tabular}{ll|l|l|l|ll}
\hline$R \$$ & 0,18 & $R \$ 0,02$ & $R \$ 0,06$ & $R \$ 0,46$ \\
\hline & 0,18 & $R \$ 0,02$ & R 0,06 & R 0,48
\end{tabular} \begin{tabular}{|ll|ll|ll|ll|}
\hline$R \$$ & 0,18 & $R \$ 0,02$ & $R \$ 0,06$ & $R \$ 0,48$ \\
\hline$R$ & 0,18 & $R$ & 0,02 & R 0,06 & R 0,47 \\
\hline
\end{tabular} \begin{tabular}{|ll|ll|ll|ll|}
\hline$R \$$ & 0,18 & $R \$ 0,02$ & $R \$ 0,06$ & $R \$ 0,47$ \\
\hline
\end{tabular} \begin{tabular}{|ll|ll|ll|ll}
\hline$R \$$ & 0,18 & $R \$ 0,02$ & $R \$ 0,06$ & $R \$ 0,59$ \\
\hline & 0,18 & $R \$ 0,02$ & $R \$ 0,06$ & $R \$ 0,33$ \\
\hline
\end{tabular} \begin{tabular}{|ll|l|l|l|l|}
\hline$R \$$ & 0,18 & $R \$ 0,02$ & $R \$ 0,06$ & $R \$ 0,33$ \\
\hline$R$ & 0,18 & $R \$ 0,02$ & $R \$ 0,06$ & $R$ & 0,49 \\
\hline
\end{tabular} \begin{tabular}{ll|ll|llll}
$\mathrm{R} \$$ & 0,18 & $\mathrm{R} \$ 0,02$ & $\mathrm{R} \$ 0,06$ & $\mathrm{R} \$ 0,49$ \\
\hline
\end{tabular} \begin{tabular}{|ll|l|l|l|l|}
\hline$R \$$ & 0,18 & $R \$ 0,02$ & $R \$ 0,06$ & $R \$ 0,68$ \\
\hline
\end{tabular}

\begin{tabular}{|ll|l|l|l|l|}
\hline$R \$$ & 0,09 & $R \$ 0,02$ & $R \$ 0,03$ & $R \$ 0,36$ \\
\hline
\end{tabular} \begin{tabular}{|ll|l|l|l|l|l|}
\hline$R$ & 0,09 & $R \$ 0,02$ & $R \$ 0,03$ & $R \$ 0,31$ \\
\hline
\end{tabular} \begin{tabular}{|ll|l|l|l|l|}
\hline$R \$$ & 0,09 & $R \$ 0,02$ & $R \$ 0,03$ & $R \$ 0,21$ \\
\hline
\end{tabular} \begin{tabular}{|ll|l|l|l|l|}
\hline$R \$$ & 0,09 & $R \$ 0,02$ & $R \$ 0,03$ & $R \$ 0,28$ \\
\hline
\end{tabular}

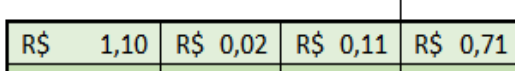
\begin{tabular}{|ll|l|l|ll|l|}
\hline$R \$$ & 1,10 & $R \$$ & 0,02 & $R \$ 0,11$ & $R \$ 0,64$ \\
\hline
\end{tabular}

\section{Quadro 6. Custos por Etapa}

Fonte: Dados Primários. 
Observa-se no Quadro 06, que os custos foram analisados em todas as etapas do processo, diretas e indiretas. Os insumos como: viés, elástico, cadarço e cordão foram analisados pela metragem utilizada por produto, sendo que a linha foi empregada a quantidade que um carretel consegue produzir unidades em relação ao seu custo. $O$ tecido era calculado pelo seu custo por metro quadrada, entretanto, isso não considera as sobras do corte e nem a maneira que o tecido é cortado na mesa de enfesto, tendo em vista que alguns cortes de produtos têm mais material descartado do que outros, o preço por $\mathrm{m}^{2}$ não condiz com a quantidade de tecido que seja realmente utilizada, estimando um custo com tecidos e uma produção menor do que o valor real.

O método adotado para avaliação dos custos do tecido se baseia na observação da maneira que os produtos são cortados, em alguns casos, os tamanhos diferentes de produtos são cortados juntos para diminuir os retalhos, um exemplo disso são os forros, o tamanho $50 \times 1,10 \mathrm{~cm}$ é cortado na vertical, com 2 unidades de $50 \times 1,10 \mathrm{~cm}$ e uma de $35 \times 1,10 \mathrm{~cm}$, uma no lado da outra, aproveitando o máximo da largura do tecido de que possui $1,40 \mathrm{~cm}$, tendo uma perda estimada de $5 \times 1,10 \mathrm{~cm}$ a cada 3 forros cortados, um valor que através do novo método de custeio passa a ser considerado nos custos.

A produção diária varia de acordo com o desgaste físico dos colaboradores do departamento de costura, devido ao estudo ser realizado em uma empresa com poucos operários, em uma área que a MOD é totalmente correlacionada com a quantidade produzida, notou-se que a produção varia significantemente de acordo com o dia da semana e o desgaste dos colaboradores.

Constatou-se uma variação na produção ao analisar alguns produtos na mesma etapa do processo em dias alternados. Um exemplo mais prático é a relação entre a produção com a entrada de novos pedidos. Assim que chegam novos pedidos são encaminhados para o setor de embalagem, onde os itens solicitados são baixados dos estoques para a expedição, caso tenha algum item que a quantidade em estoque não seja suficiente para suprir o pedido é gerado uma ordem de produção com a quantidade aproximada de itens faltantes. Assim, no término do corte os itens são enviados para o setor de costura. Nesse momento, percebeu-se uma agilidade maior entre os processos, diferentemente do que foi analisado quando a produção é destinada para 0 estoque. 
Averiguado os custos, constatou-se que os preços por categoria variam aproximadamente $17 \%$ nas capas para máquina de lavar, em valores $R \$ 14,42$ a $R \$$ 16,87 , nos forros para passar roupas se obteve uma variação de quase $70 \%$, em valores $R \$ 6,28$ a $R \$ 10,64$, os protetores de roupa foi onde teve a maior variação de $117 \%$, em valor $R \$ 2,08$ a $R \$ 4,53$, já as almofadas para passar tiveram uma variação de aproximadamente $12 \%$, em valores $R \$ 9,90$ a $R \$ 11,24$.

\section{CONSIDERAÇÕES FINAIS}

O objetivo do estudo consistiu em analisar a capacidade produtiva com base nos custos em uma pequena indústria de confecção, mensurando a quantidade máxima de produção.

Através de uma análise mais apurada dos custos, a empresa tem a possibilidade de identificar melhor sua linha de produção, encontrando maneiras de torná-la mais ágil. Dos setores analisados, o maior gargalo de produção é o setor de costura, devido a ser um processo que ainda é grande parte feita manualmente pelas costureiras, isto é um dos pontos que podem ser melhorados.

Com um maior domínio dos custos, tem-se uma maior visibilidade de margem de contribuição, uma melhor confiabilidade nos custos unitários, na precificação dos produtos, identificando possíveis itens que podem estar puxando a receita para baixo por má precificação.

Ao analisar a capacidade produtiva diária, comparando um setor com o outro, pode-se dizer que na categoria capa de máquina, o setor de corte consegue produzir uma média 3 vezes mais do que o setor de costura; em relação ao forro para tábua, é uma média de 6 vezes mais do que o setor de costura, nos protetores chega a 5 vezes mais produção do que o setor de corte em relação ao de costura, na categoria almofada para roupa, é o que apesenta a menor diferença 2,4 vezes.

Como sugestões para pesquisas futuras, os resultados observados demonstram maior necessidade de detalhamento de quais partes e produtos do processo podem ser terceirizados para um aumento de produção, em períodos de grande demanda ou para 
estudar a ampliação do departamento. A análise do processo de forma completa e estudos que analisem a capacidade produtiva é importante para o enriquecimento da contabilidade de custos.

A limitação da pesquisa consiste na escolha metodológica e da análise utilizada. Em razão de se tratar de um estudo de caso, não há a possibilidade de generalizar os resultados obtidos.

\section{REFERÊNCIAS}

Abbas, K., Gonçalves, M. N., \& Leoncine, M. (2012). Os métodos de custeio: vantagens, desvantagens e sua aplicabilidade nos diversos tipos de organizações apresentadas pela literatura. Contexto, v. 12, n. 22, p. 145-159. Recuperado de: https://seer.ufrgs.br/ConTexto/article/view/33487 Acesso em: 10/Jul./2020.

Associação Brasileira da Indústria Têxtil e de Confecção (Brasil). (2020). O Poder da Moda. Recuperado de: <https://www.abit.org.br>. Acesso em: 23/ago./2020.

Alonso, M. (1999). Custos no serviço público. Revista do Serviço Público, v. 50, n. 1, p. 37-63.

Araújo, A. M. C. (2002). Redes de subcontratação e trabalho a domicílio na indústria de confecção: um estudo na região de Campinas. Cadernos Pagu, n. 17-18, p. 267310. Recuperado de: https://www.scielo.br/scielo.php?pid=S010483332002000100010\&script=sci abstract\&tlng=pt Acesso em: 15/jul./2020.

Barsanti, H. G. (2016). Método de custeio ABC aplicado: uma pesquisa bibliométrica. (Trabalho de Conclusão de Curso). Ciências Contábeis, Universidade Federal do Rio Grande do Sul, UFRGS, Porto Alegre, Rio Grande do Sul.

Boff, C. P. (2013). Estudo do Gerenciamento da Capacidade Produtiva na empresa Isca Tecnologias LTDA. (Trabalho de Conclusão de Curso). Administração. Universidade Regional do Noroeste do Estado do Rio Grande do Sul, Unijuí, ljuí, Rio Grande do Sul, Recuperado de: https://bibliodigital.unijui.edu.br:8443/xmlui/bitstream/handle/123456789/2103/TCC \%20-\%20Carolina\%20Patias\%20Boff.pdf?sequence=1 Acesso em: 10/mai./2020.

Braga, E. C. (2009). Gestão de Custos através do Custeio Baseado em Atividades (ABC): um estudo de caso em um projeto de desenvolvimento de software. (Dissertação de Mestrado). Ciências Contábeis. Universidade Estadual do Rio de Janeiro, UERJ, Rio de Janeiro. 
Coronetti, J., Beuren, I. M., \& De Sousa, M. A. B. (2003). Os métodos de custeio utilizados nas maiores indústrias de Santa Catarina. In: Anais do Congresso Brasileiro de Custos-ABC. Recuperado de: https://anaiscbc.emnuvens.com.br/anais/article/view/2560 Acesso em: 10/mai./2020.

Derbeck, E., \& Nagy, C. (2001). Contabilidade de custos. (11a ed.). São Paulo: Thomson.

Koliver, O. (2009). Contabilidade de Custos. Curitiba: Juruá.

Leão, T. (2019). O que é gargalo de produção? Recuperado de: $<$ https://www.nomus.com.br/blog-industrial/gargalo-de-producao/s. Acesso em: 24/abr./2020.

Martins, E. (2010). Contabilidade de custos. (10a ed.). São Paulo: Atlas.

Oliveira, L. M. de., \& Peres Junior, J. H. (2000). Contabilidade de custos. São Paulo: Atlas.

Rabelo, E. C., Borgert, A., \& De Medeiros, C. S. C. (2011). Apropriação dos custos indiretos de fabricação em indústrias cerâmicas do Sul Catarinense. ABCustos, v. 6, n. 3 2011. Recuperado de: https://abcustos.emnuvens.com.br/abcustos/article/view/155 Acesso em: 10/jul./2020.

Raupp, F. M., \& Beuren, I. M. (2014). Metodologias de custos utilizadas nas maiores indústrias do setor têxtil do Estado de Santa Catarina. Revista Ciências Administrativas ou Journal of Administrative Sciences, v. 17, n. 3. Recuperado de: https://periodicos.unifor.br/rca/article/view/3299. Acesso em: 15/Mai./2020.

Sá, C. A. (2011). O método de custeio por absorção e o método de custeio variável. Recuperado de: <http://carlosalexandresa.com.br/artigos/O-Metodo-de-Custeio-porAbsorcao-e-o-Metodo-de-Custeio-Variavel.pdf > Acesso em 04/abr./2020.

Santos, M. A. dos. (2018). Contabilidade de custos. (1a ed.). Salvador

Data de Submissão: 15/09/2020

Data de Aceite: 24/08/2021

ANEXOS - Fonte: TCE Proposta de um sistema logístico para o comércio eletrônico da empresa (2013). 


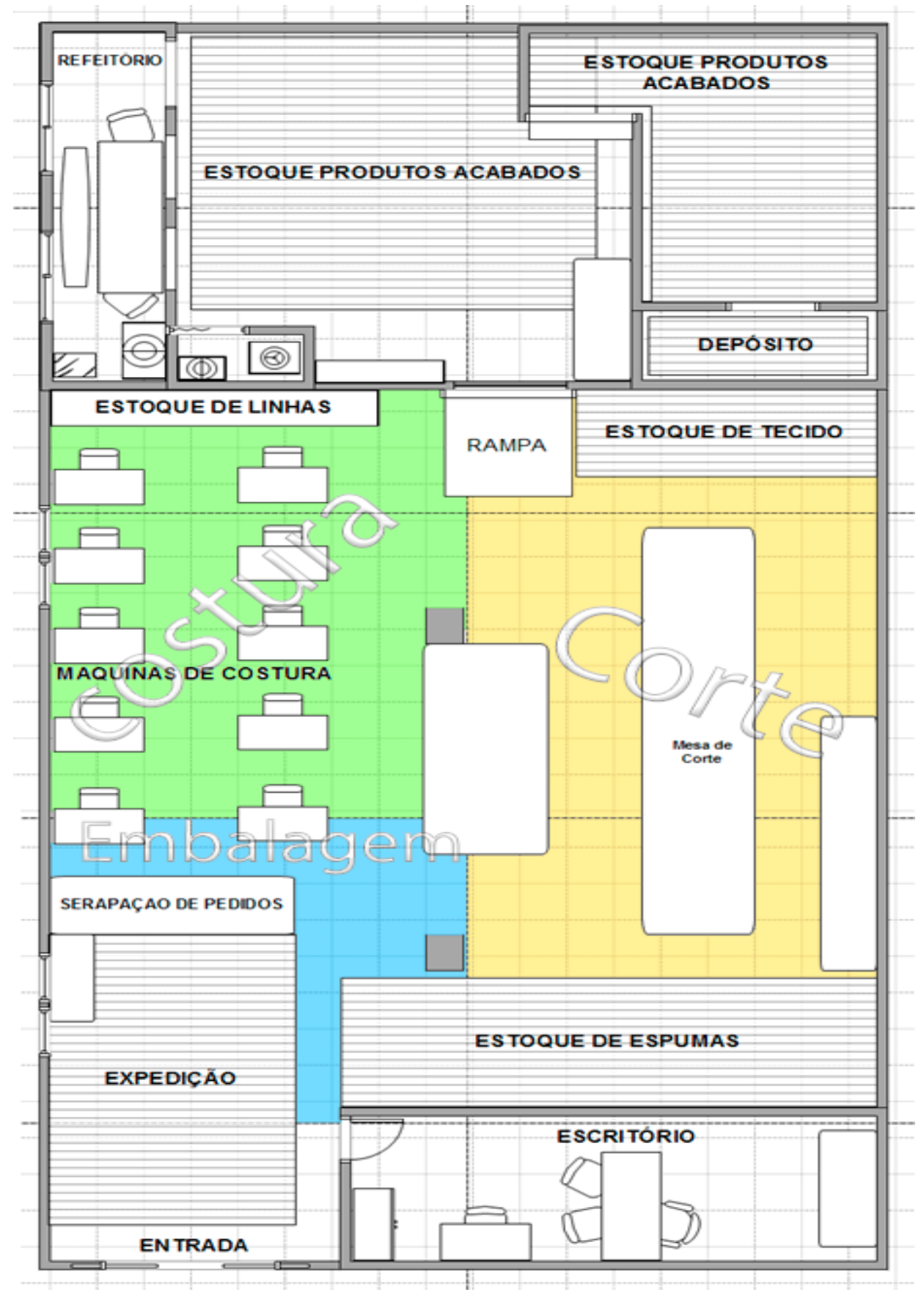

